\title{
Treatable Leukoencephalopathy in a Patient with Hypophosphatemia
}

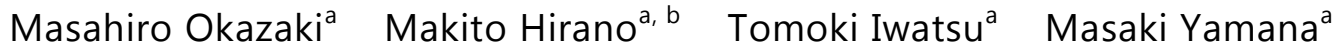 \\ Hidekazu Suzuki $^{\mathrm{a}}$ Takao Satou $^{c}$ Susumu Kusunoki ${ }^{\mathrm{a}}$ \\ a Department of Neurology, Kindai University Faculty of Medicine, Osakasayama, Japan; \\ ${ }^{b}$ Department of Neurology, Sakai Hospital Kindai University Faculty of Medicine, \\ Sakai, Japan; 'Department of Pathology, Kindai University Faculty of Medicine, \\ Osakasayama, Japan
}

\section{Keywords}

Leukoencephalopathy · Hypophosphatemia - Decreased phosphate level - Phosphate binder . Osmotic myelinolysis

\begin{abstract}
We report the first patient with pathologically proven leukoencephalopathy associated with hypophosphatemia. A 61-year-old woman had repetitive episodes of decreased consciousness with pontine and pallidal lesions and extensive leukoencephalopathy on MRI, later found to be associated with hypophosphatemia. Although hypophosphatemia has been linked to central pontine and extrapontine myelinolysis (osmotic myelinolysis), lesions in the deep white matter have not been reported. Brain biopsy performed during the first diagnosis process revealed nonspecific demyelination with gliosis, a finding similar to that of chronic osmotic myelinolysis. After normalization of phosphate levels, her consciousness completely improved and MRI abnormalities partly resolved. We should consider that leukoencephalopathy can be associated with hypophosphatemia, which is often treatable.
\end{abstract}




\section{Introduction}

Hypophosphatemia is a critical condition that ultimately reduces a primary energy source, ATP. Thus, it affects virtually all organs and tissues [1]. In nervous systems, hypophosphatemia causes irritability, decreased consciousness, and seizure [1]. Reports about brain imaging abnormality have been limited, with no pathological finding reported. The aforementioned symptoms are nonspecifically seen and do not readily lead to the correct diagnosis, which is even more difficult when symptoms in the other organs, such as cardiovascular or respiratory failure, are not apparent. The diagnosis is especially difficult in patients with chronic renal failure, since they are at risk for an increase, but not a decrease, in serum phosphate levels [2]. We report the first patient with pathologically proven leukoencephalopathy associated with hypophosphatemia.

\section{Case Report}

A 61-year-old Japanese woman had two episodes of decreased consciousness with MRI abnormalities. The first episode started at the age of 55 years. Her level of activity of daily living gradually decreased over 2 months. She was then admitted to a local hospital. She had a history of diabetic retinopathy at the age of 51 years (the onset age of diabetes mellitus was unclear), and then diabetic nephropathy, starting hemodialysis three times a week since the age of 52 years. She received subcutaneous insulin injections, which had been stopped 4 months before the first episode but were restarted after admission to our hospital. She also received gastrointestinal medication, but had not received phosphate binders or magnesium. She also had a history of left breast cancer, which was completely resected by operation at the age of 54 years, without recurrence so far. No alcoholism was noted. Because her consciousness was gradually disturbed, 24 days later, she was transferred to our hospital. On admission, her body temperature was $36.2^{\circ} \mathrm{C}$. Her blood pressure was $162 / 82$ with a heart rate of 90 beats/min, and 2 days later, it decreased to 138/77 with a heart rate of 90 beats/min without antihypertensive medication. Routine blood tests revealed increased levels of creatinine $(4.81 \mathrm{mg} / \mathrm{dL}$, normal $0.46-0.79 \mathrm{mg} / \mathrm{dL})$, deceased levels of total protein (6.4 mg/dL, normal 6.6-8.1 mg/dL) and albumin $(3.2 \mathrm{mg} / \mathrm{dL}$, normal 4.1-5.1 mg/dL), but normal levels of hemoglobin $A_{1 c}$, blood nitrogen, sodium, calcium, potassium, and chloride. Cerebrospinal fluid (CSF) contained $1 / 3$ mononuclear cell/ $\mu \mathrm{L}$ (normal $\leq 5$ ), $148 \mathrm{mg} / \mathrm{dL}$ of protein (normal $\leq 50 \mathrm{mg} / \mathrm{dL}$ ), but no oligoclonal IgG bands. IgG index was normal. Brain MRI showed T2-high lesions in the pons and the bilateral globus pallidus and deep white matters, i.e., leukoencephalopathy (Fig. 1). Diffusion-weighted image showed very mild high signals in the white matter lesions without decreased signal on apparent diffusion coefficient map (not shown), indicative of T2 shine-through (no true diffusion restriction) [3]. Her consciousness level decreased to deep coma. She then received intravenous hyperalimentation treatment. Brain biopsy was performed from the right frontal lobe. The result revealed nonspecific demyelination with gliosis in the deep white matter (Fig. 2). No tumor cells or infiltrated inflammatory cells were evident. However, inflammatory leukoencephalitis such as acute disseminated encephalomyelitis was not completely excluded, and three cycles of methylprednisolone pulse therapy were given. Her consciousness and leukoencephalopathy resolved thereafter (Fig. 1), without apparent changes of pontine lesions. She became able to ambulate and was discharged from our hospital 111 days after admission to our hospital. 
The second episode occurred 6 years later. She fell down and had a fracture of lumbar vertebrae at the age of 61 years. She was admitted to a local hospital of orthopedic surgery. She already received precipitated calcium carbonate $(1,000 \mathrm{mg} /$ day $)$ and lanthanum carbonate $(500 \mathrm{mg} /$ day), phosphate binders to prevent high phosphate levels by hemodialysis, and subcutaneous insulin injections. After 1 month, she had decreased consciousness (E3V1M4 on the Glasgow Coma Scale). She was then transferred to our hospital (day 1). On admission, body temperature was $36.3^{\circ} \mathrm{C}$. Her blood pressure was $166 / 87$ with a heart rate of 78 beats/min, and 2 days later, it decreased to 128/70 with a heart rate of 76 beats/min with administration of $1.25 \mathrm{mg} /$ day of carvedilol. Respiratory disturbances were not apparent. Deep tendon reflexes were absent in all limbs with bilateral extensor plantar responses. Her consciousness gradually worsened to deep coma (E1V1M4) on day 9. Brain MRI showed new T2-high lesions in the pons and the bilateral globus pallidus and cerebral white matters (Fig. 1), with a diffusion-weighted imaging finding similar to that in the first hospital stay. The electroencephalogram showed diffuse slow waves. Routine blood tests showed increased levels of blood nitrogen $(45 \mathrm{mg} / \mathrm{dL})$, creatinine $(8.26 \mathrm{mg} / \mathrm{dL})$, and hemoglobin $\mathrm{A}_{1 \mathrm{c}}$ (6.7\%), decreased levels of chloride ( $97 \mathrm{mEq} / \mathrm{L})$ and platelets $(11.1 / \mu \mathrm{L})$, but normal levels of sodium, calcium, potassium, and ammonia. A blood gas test in room air revealed very mild metabolic alkalosis without evidence of respiratory failure ( $\mathrm{pH} 7.46$ [normal, 7.35-7.45]; $\mathrm{PaCO}_{2} 39.1$ Torr; $\mathrm{PaO}_{2} 89.4$ Torr; $\mathrm{HCO}^{3-} 27.2 \mathrm{mEq} / \mathrm{L}$ [normal, $22-26 \mathrm{mEq} / \mathrm{L}$ ]; actual base excess +3.3 [normal -2 to +3 ]; standard base excess +3.4 [normal -3 to +2 ]; and $\mathrm{SaO}_{2}$ 95.2\%). CSF contained 1 mononuclear cell/ $\mu \mathrm{L}$ (normal $\leq 5$ ), $149 \mathrm{mg} / \mathrm{dL}$ of protein (normal $\leq 50 \mathrm{mg} / \mathrm{dL}$ ), and a normal level of myelin basic protein, but no oligoclonal IgG bands. IgG index was normal. To exclude paraneoplastic syndrome including recurrence of breast cancer or other types of malignancy, whole-body computed tomography was performed, but obtained negative results. Three cycles of methylprednisolone pulse therapy mildly improved her consciousness with considerable fluctuation (E1V1M4 to E4V1M4).

We explored the reason of her decreased consciousness, and then found a low level of phosphate $(1.8 \mathrm{mg} / \mathrm{dL}$, normal $2.7-4.6 \mathrm{mg} / \mathrm{dL})$, which decreased to $1.0 \mathrm{mg} / \mathrm{dL}$ during the second hospital stay. The activated vitamin $\mathrm{D}\left(1,25(\mathrm{OH})_{2}\right.$-vitamin $\left.\mathrm{D}\right)$ level decreased to 4.7 $\mathrm{pg} / \mathrm{mL}$ (normal $20.0-60.0 \mathrm{pg} / \mathrm{mL}$ ), while the parathyroid hormone level increased to more than $3,200 \mathrm{pg} / \mathrm{mL}$ (normal $160-520 \mathrm{pg} / \mathrm{mL}$ ). FGF23 also increased to $3,700 \mathrm{pg} / \mathrm{mL}$ (normal $14-68 \mathrm{pg} / \mathrm{mL})$.

Intravenous sodium phosphate infusion $(20 \mathrm{mmol} /$ day $)$ was started with discontinuation of phosphate binders, which gradually improved her consciousness within 1 month. She then became able to ambulate. The protein level in CSF decreased to $75 \mathrm{mg} / \mathrm{dL}$. Brain MRI also showed decreased pontine, pallidal, and white matter lesions, especially in the bilateral occipital regions (Fig. 1). She was discharged from our hospital 47 days after the start of phosphate complementation.

We retrospectively reviewed phosphate levels during the first stay in our hospital. Hypophosphatemia $(1.2 \mathrm{mg} / \mathrm{dL})$ was found on the seventh day, but it resolved $(3.0 \mathrm{mg} / \mathrm{dL}) 3$ days later with no specific treatment. Her decreased consciousness remained unchanged for a further 6 days, when brain biopsy was performed.

\section{Discussion}

We report a patient who had pontine and pallidal lesions with leukoencephalopathy, which was later found to be associated with hypophosphatemia. Leukoencephalopathy has 
been caused by toxic, acquired vascular, metabolic, and infectious/inflammatory insults, as well as inherited disorders [4]. Two reports described patients with hypophosphatemia who had central pontine and extrapontine myelinolysis (osmotic myelinolysis) [5, 6]. These patients did not have leukoencephalopathy or lesions in the cerebral white matter, unlike our patient. However, extrapontine myelinolysis caused by other conditions such as the rapid correction of chronic hyponatremia have been reported to involve the cerebral white matter [7]. Thus, leukoencephalopathy in our patient can be possibly explained by a mechanism similar to that of osmotic myelinolysis.

We described the first pathological finding of hypophosphatemia-associated myelinolysis: nonspecific demyelination with gliosis (proliferation of astrocytes). A review of pathology in 58 patients with hyponatremia-related osmotic myelinolysis described no or little gliosis [8]. However, several other reports demonstrated the presence of gliosis, especially in patients with chronic courses $[9,10]$. In contrast to patients with rapid correction of hyponatremia, those with hypophosphatemia had unclear onset of decreased phosphate levels, suggestive of more chronic courses in hypophosphatemia-related osmotic myelinolysis.

The MRI findings of our patient had atypical as well as typical features for osmotic myelinolysis. Atypical features included the involvement of the deep white matter, a region affected in leukoencephalopathy. The aforementioned review of pathology of osmotic myelinolysis described that the preferentially involved region of white matter was the cerebral subcortex but not the deep white matter, with no explainable reason [8]. The shape of the pontine lesion in our patient, a diffuse unclear shape, is another atypical feature, since most reported patients with osmotic myelinolysis had a clear round or triangular shape. However, a similar diffuse pattern has been reported in a patient with hypophosphatemia-related osmotic myelinolysis [11]. On the other hand, our patient had typical MRI findings in the bilateral globus pallidus for osmotic myelinolysis [12].

The alternative diagnosis of recurrent/transient leukoencephalopathy included posterior reversible encephalopathy syndrome (PRES), because on the first and second admissions, our patient had renal failure and hypertension, which are risk factors of PRES [13]. However, her pathological finding differed from typical findings of PRES: dilated perivascular spaces containing proteinaceous exudates and macrophages with fibrinoid necrosis and acute hemorrhage [14]. Another alternative diagnosis included immunological diseases, such as acute disseminated encephalomyelitis (ADEM). This diagnosis may be supported by the observed response to methylprednisolone pulse therapy. A further supported finding was the increased CSF protein level, a finding frequently observed in ADEM [15] but rarely in hypophosphatemia-related osmotic myelinolysis [5]. Nonetheless, a reported patient with severe hypophosphatemia had a mildly increased CSF protein level $(94 \mathrm{mg} / \mathrm{dL}$ ) [16]. In addition, the typical pathological features of ADEM are perivascular infiltrates mostly of foamy macrophages [17], a finding that was absent in our patient. We thus speculate that the primary factor causative for the white matter lesions is attributed to hypophosphatemia-related osmotic myelinolysis, but that some other factors, such as immunological factors, are necessary for affecting the deep white matter.

Elucidation of the mechanism underlying hypophosphatemia was important to prevent recurrence. Hypophosphatemia has been associated with various mechanisms, which are divided into three categories [1]: malabsorption from the intestine, transport of phosphate into cells, and increased urinary excretion. The first mechanism includes malnutrition, alcoholism, decreased level of activated vitamin $\mathrm{D}$, and drugs such as magnesium and phosphate binders. Our patient had decreased levels of activated vitamin D probably due to chronic renal failure, and received phosphate binders during the second hospital stay. The observed 
increased level of FGF23 may also have reduced the activated vitamin D level [18]. The second mechanism includes respiratory alkalosis and insulin treatment. She received insulin treatment during the second hospital stay. The third mechanism includes hyperparathyroidism and drugs (corticosteroids and diuretics), but it is not applicable in our patient, since she had no or little urine for phosphate to be excreted. In the first hospital stay, hypophosphatemia was resolved without a specific infusion of sodium phosphate. One reason may include that she received relatively phosphate-rich intravenous hyperalimentation, unlike in the second hospital stay, where she received phosphate binders through a nasogastric tube. Collectively, our patient had several reasons for hypophosphatemia.

In conclusion, although leukoencephalopathy is associated with various causes, we should consider that it can be associated with hypophosphatemia. Why the phosphate levels did not parallel her consciousness levels or MRI findings remains to be elucidated, but hypophosphatemia might have triggered myelinolysis, the symptoms of which lasted after the correction of phosphate levels. Similar remaining symptoms after normalization of the phosphate level have been reported in a patient with hypophosphatemia-associated osmotic myelinolysis [6]. We also cannot completely deny the coincidental occurrence of hypophosphatemia and leukoencephalopathy, and therefore, accumulation of cases is apparently needed to draw firm conclusion of their link.

\section{Statement of Ethics}

The authors have no ethical conflicts to declare.

\section{Disclosure Statement}

The authors have no conflicts of interest to disclose.

\section{References}

1 Amanzadeh J, Reilly RF Jr: Hypophosphatemia: an evidence-based approach to its clinical consequences and management. Nat Clin Pract Nephrol 2006;2:136-148.

-2 Pohlmeier R, Vienken J: Phosphate removal and hemodialysis conditions. Kidney Int Suppl 2001;78:S190-S194.

3 Roberts TP, Rowley HA: Diffusion weighted magnetic resonance imaging in stroke. Eur J Radiol 2003;45:185-194.

4 Vanderver A, Prust M, Tonduti D, Mochel F, Hussey HM, Helman G, Garbern J, Eichler F, Labauge P, Aubourg P, Rodriguez D, Patterson MC, Van Hove JL, Schmidt J, Wolf NI, Boespflug-Tanguy O, Schiffmann R, van der Knaap MS: Case definition and classification of leukodystrophies and leukoencephalopathies. Mol Genet Metab 2015;114:494-500.

5 Qadir F, Hasan A, Masood M: Extra pontine myelinolysis associated with hypophosphatemia. J Pak Med Assoc 2005;55:254-256

6 Yamashita C, Shigeto H, Maeda N, Torii T, Ohyagi Y, Kira J: A case of central pontine myelinolysis caused by hypophosphatemia secondary to refeeding syndrome. Case Rep Neurol 2015;7:196-203.

7 Chua GC, Sitoh YY, Lim CC, Chua HC, Ng PY: MRI findings in osmotic myelinolysis. Clin Radiol 2002;57:800-806.

8 Gocht A, Colmant HJ: Central pontine and extrapontine myelinolysis: a report of 58 cases. Clin Neuropathol 1987;6:262-270.

Wright DG, Laureno R, Victor M: Pontine and extrapontine myelinolysis. Brain 1979;102:361-385. Hornik A, Rodriguez Porcel FJ, Agha C, Flaster M, Morales Vidal S, Schneck MJ, Lee J, Biller J: Central and extrapontine myelinolysis affecting the brain and spinal cord. An unusual presentation of pancreatic encephalopathy. Front Neurol 2012;3:135. 
11 Falcone N, Compagnoni A, Meschini C, Perrone C, Nappo A: Central pontine myelinolysis induced by hypophosphatemia following Wernicke's encephalopathy. Neurol Sci 2004;24:407-410.

12 Lim CC: Magnetic resonance imaging findings in bilateral basal ganglia lesions. Ann Acad Med Singapore 2009;38:795-798.

13 Hobson EV, Craven I, Blank SC: Posterior reversible encephalopathy syndrome: a truly treatable neurologic illness. Perit Dial Int 2012;32:590-594.

14 Kheir JN, Lawlor MW, Ahn ES, Lehmann L, Riviello JJ, Silvera VM, McManus M, Folkerth RD: Neuropathology of a fatal case of posterior reversible encephalopathy syndrome. Pediatr Dev Pathol 2010;13:397-403.

15 Schwarz S, Mohr A, Knauth M, Wildemann B, Storch-Hagenlocher B: Acute disseminated encephalomyelitis: a follow-up study of 40 adult patients. Neurology 2001;56:1313-1318.

16 Becker S, Dam G, Hvas CL: Refeeding encephalopathy in a patient with severe hypophosphataemia and hyperammonaemia. Eur J Clin Nutr 2015;69:279-281.

17 Kuhlmann T, Lassmann H, Bruck W: Diagnosis of inflammatory demyelination in biopsy specimens: a practical approach. Acta Neuropathol 2008;115:275-287.

18 Quarles LD: Role of FGF23 in vitamin D and phosphate metabolism: implications in chronic kidney disease. Exp Cell Res 2012;318:1040-1048. 


\section{Case Reports in Neurology}

First hospital stay
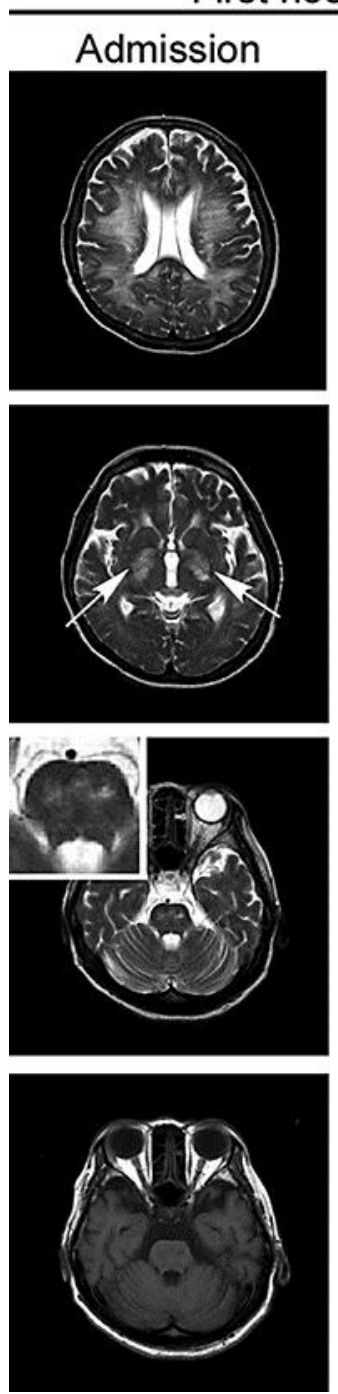

Discharge
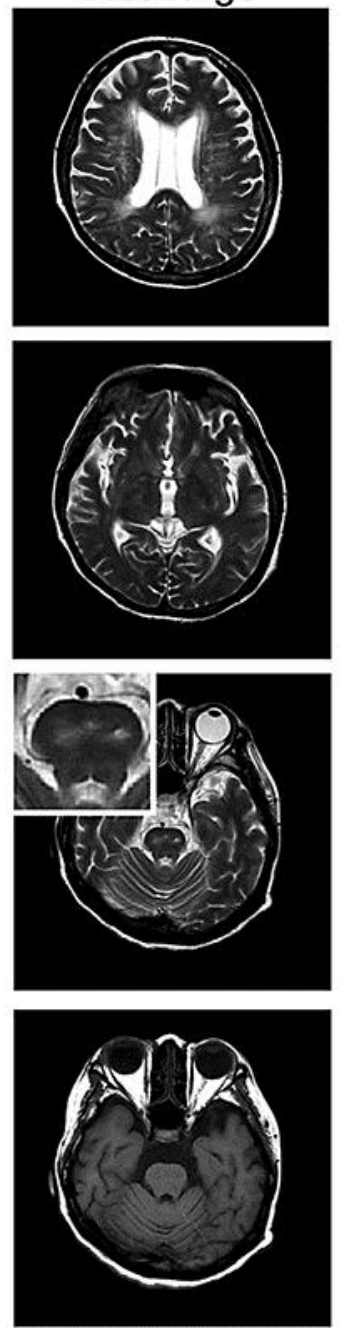

Second hospital stay
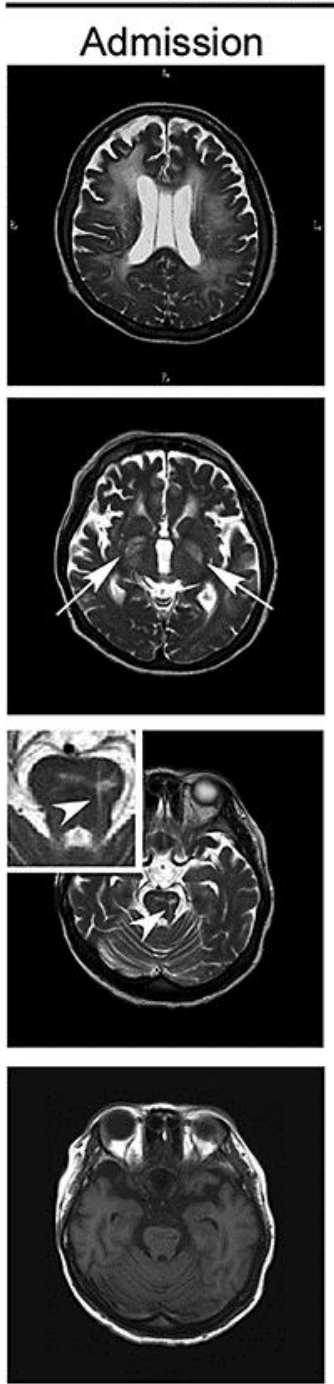
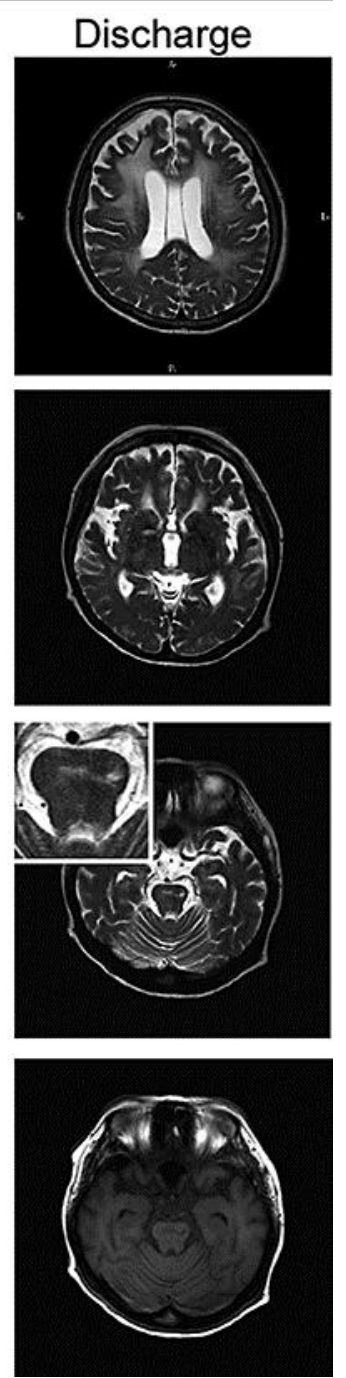

Fig. 1. Series of T2-weighted images (1st-3rd rows, TR/TE $=4,200 / 100 \mathrm{~ms}$, Philips MRI 1.5T) and of T1weighted images ( 4 th row, TR/TE $=527 / 10$ ) of MRI. First column: images on the first admission showed severe leukoencephalopathy with pallidal (arrow) and pontine lesions. Second column: images at the first discharge from our hospital showed marked decreases of T2-high signals in the white matter and globus pallidus, but similar signals in the pons. Third column: images on the second admission showed a finding similar to those on the first admission, with a new lesion in the pons (arrowhead). Fourth column: images at the second discharge from our hospital showed mild decreases of T2-high signals in the white matter of the occipital lobes and apparent decreases of signals in the globus pallidus and pons. 


\section{Case Reports in Neurology}
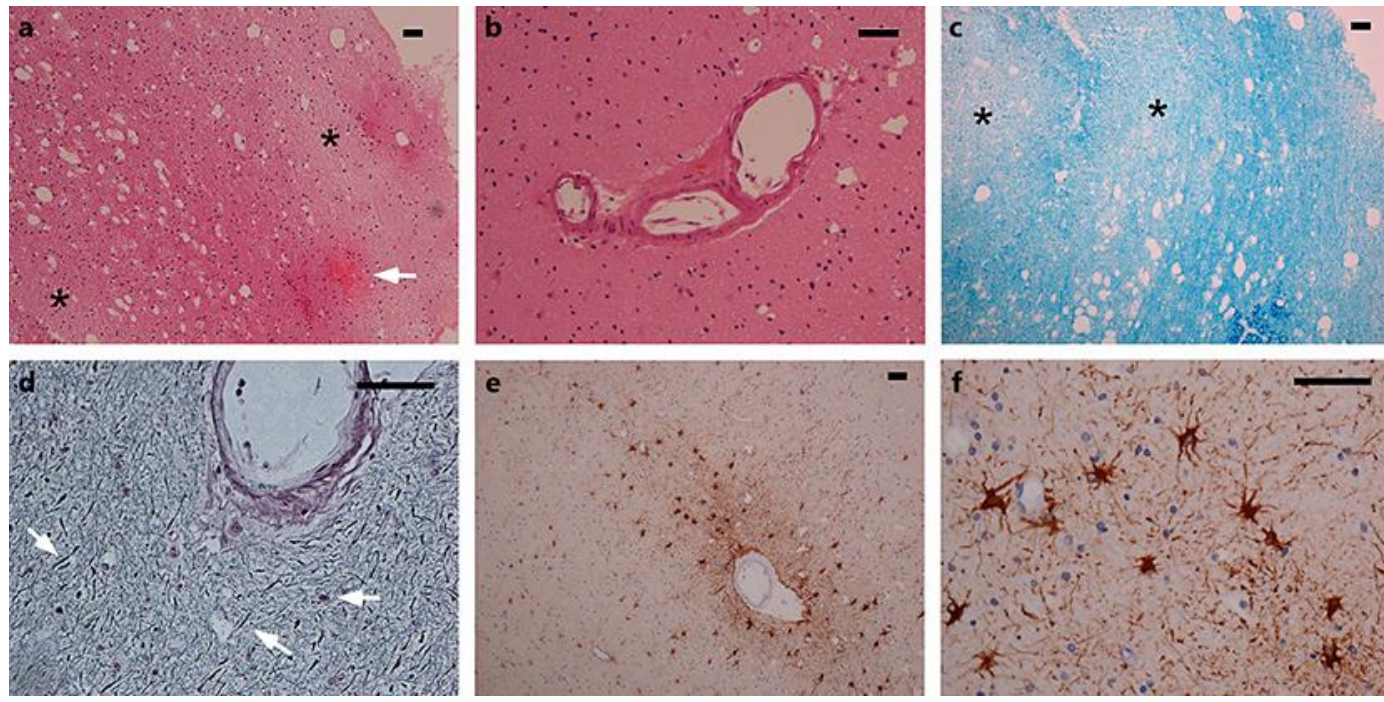

Fig. 2. a Hematoxylin and eosin (HE) staining showed probable demyelination $\left({ }^{*}\right)$, but no tumor cells or infiltrated inflammatory cells in the white matter. Possible artificial bleeding was present on HE staining (arrow). b No infiltration of inflammatory cells around vessels. c Luxol fast blue staining of the brain specimen from the right frontal lobe biopsied during the first admission confirmed demyelination $(*)$ ). $\mathbf{d}$ Bodian staining showed relative preservation of axons (arrows). e Immunostaining with anti-glial fibrillary acidic protein antibody revealed an increase of astrocytes. $f$ High magnification showed that astrocytes enlarged with long cell processes, indicating gliosis. Bar represents $50 \mu \mathrm{m}$. 